$\mathrm{M}$ igration ist ein Phänomen, das seit Anbeginn der Menschheit existiert. Ende des 20. Jahrhunderts fand eine starke Welle von Migration statt, die auch Deutschland betraf. So wurden durch Anwerbungen z.B. aus Italien, Spanien, dem ehemaligen Jugoslawien und der Türkei Arbeitsmigranten nach Deutschland geholt. Inzwischen leben neben diesen Arbeitsmigranten erster, zweiter, dritter und vierter Generation auch aus Osteuropa stammende Aussiedlerpopulationen, Bürgerkriegsflüchtlinge, Asylantragsteller und traumatisierte Flüchtlinge sowie Menschen ohne Aufenthaltsstatus hier. Ihr Anteil an der Gesamtbevölkerung beträgt knapp 9\%. Somit besteht in Deutschland eine multikulturelle Gesellschaft.

Infolge der unterschiedlichen kulturellen Besonderheiten wäre es nachvollziehbar, dass auch unterschiedliche Beschwerden bzw. Symptome und Syndrome zu erwarten wären. Bei den eigentlichen psychiatrischen Krankheitsbildern sind die Symptome kulturübergreifend, die Bedeutung der einzelnen Symptome und der Umgang mit ihnen ist hingegen kulturgebunden. Neben den kulturübergreifenden Symptomen bzw. Syndromen können auch kulturspezifische Syndrome, die nur in einer spezifischen kulturellen Umgebung vorkommen, beobachtet werden.

Nationale und internationale Untersuchungen haben aufgezeigt, dass die Inanspruchnahme psychiatrischer Einrichtungen durch Migranten deutlich niedriger liegt als im Vergleich zu der einheimischen Bevölkerung. Die Gründe dafür sind u.a. Informationsmangel, fehlende bzw. unzureichende interkulturelle Verständigungsmöglichkeit, Stigmatisierung, Scham, Qualifikationsund Kompetenzmängel bei den Mitarbeitern der psychiatrisch-psychotherapeutischen Versorgungssysteme. Belastungsfaktoren bzw. psychosoziale Stressoren sind u.a. schwere Arbeitsbedingungen, Arbeitslosigkeit, Verarmung, schlechte Wohnverhältnisse, Auflösung von Familienverbänden, Vereinsamung, Rollenverlust und -diffusion, Erfah-

\title{
Vorwort zur Serie „Migranten“ Interkultureller Umgang mit
psychisch kranken Migranten
}

Im Rahmen dieser Serie zum interkulturellen Umgang mit psychisch kranken Migranten, die alle zwei Monate in der psychoneuro erscheinen wird, sollen Problemfelder benannt, Lösungsansätze aufgezeigt, integrative bewährte Praxismodelle vorgestellt werden und insbesondere eine Sensibilisierung erwirkt werden. Des Weiteren sollen Hilfestellungen bei der Bewältigung von interkulturellen Herausforderungen im psychiatrischen Alltag gegeben werden. Ein besonderes Anliegen besteht darin, die Besonderheiten der interkulturellen Aspekte bei psychisch kranken Migranten in das Bewusstsein der in der Psychiatrie und Psychotherapie tätigen Professionellen zu rücken.

rung von Ausgrenzung und Diskriminierung, aufenthaltsrechtliche Belastungen, Trennungserfahrung und Verlustgefühle, schlechtere Qualifikation in Schule und Beruf, schlechtere gesundheitliche Versorgung und Kommunikationsschwierigkeiten.

Nach Riedesser trafen bereits 1975 fast alle damaligen Mängel der deutschen Psychiatrie - festgestellt durch die Psychiatrieenquete - auch und in besonderem Maße bei Migranten in diagnostischen, therapeutischen und prognostischen Aspekten zu. Seiner Forderung nach mehr Studien $\mathrm{zu}$ psychopathologischen und sozialen Folgen der Arbeitsmigration wurde nur in vereinzelten Forschungsprojekten nachgegangen, obwohl seit der Psychiatrieenquete bereits 28 Jahre vergangen sind.

Das Gesundheitssystem, hier speziell in der Versorgung von seelisch kranken Migranten, ist nach wie vor unzureichend ausgestattet. Nur in wenigen Einrichtungen z.B. im Psychiatrischen Krankenhaus in Marburg oder im Psychiatrischen Zentrum Nordbaden in Wiesloch werden integrierte interkulturelle Behandlungsangebote im stationären Setting bereitgestellt. Daneben existieren einige Rehabilitationskliniken z.B. Segeberger Kliniken in Bad Segeberg, Parkland-Klinik in Bad Wildungen, Klinik am Südpark in Bad Nauheim oder die EmmaburgKlinik am Park in Bad Laaspe, die ein spezielles muttersprachliches Therapiekonzept für Migranten anbieten.
Im ambulanten Setting stellen nur wenige Einrichtungen ein entsprechendes Behandlungskonzept zur Verfügung wie das Niedersächsische Landeskrankenhaus Hildesheim, Akademisches Lehrkrankenhaus der Georg-August-Universität Göttingen. Auch an einigen anderen Einrichtungen sind in der Zwischenzeit entsprechende Spezialangebote anzutreffen. Doch sie reichen bei weitem nicht aus, um Migranten bundesweit mit dem gleichen Standard wie bei Einheimischen zu helfen. Im Sinne dieser großen Bevölkerungsgruppe sollten sich die Forschungs- und Versorgungssysteme der interkulturellen Versorgung öffnen durch Senkung der Zugangsbarrieren, Kultursensibilität und Erwerb der interkulturellen Kompetenz, Integration der transkulturellen Psychiatrie und Psychotherapie in die Aus-, Fort- und Weiterbildungssysteme.

\section{Korrespondenzadresse:}

Dr. med. Meryam Schouler-Ocak

(Vorstandsmitglied der Deutsch-Türkischen Gesellschaft für Psychiatrie, Psychotherapie und Psychosoziale Gesundheit e. V.)

Niedersächsisches Landeskrankenhaus Hildesheim

Akademisches Lehrkrankenhaus der Georg-August-Universität Göttingen Goslarsche Landstraße 60

31135 Hildesheim 